\title{
Duck $I L-2$ promoter cloning and the effects of methylation status on mRNA levels in immune tissues
}

\author{
TAO ZHANG, HEHE LIU, TIANYU ZHOU, JUN LUO, JIWEN WANG, CHUNCHUN HAN, \\ JIWEI HU, QIANFENG WU, YUSHI WANG
}

Farm Animal Genetic Resources Exploration and Innovation Key Laboratory of Sichuan Province, Sichuan Agricultural University, Chengdu, P.R. China

\begin{abstract}
Interleukin 2 (IL-2), a cytokine, plays an important role in animal immune systems. To investigate the influences of epigenetic modifications on transcription of the duck IL-2 gene, the promoter region of the duck IL-2 gene was cloned. Then, the DNA methylation status of the IL-2 gene promoter (-1337 bp/-924 bp) in immune tissues of ducks was determined using the Sequenom Mass Array methylation technique, and their corresponding expression levels were determined using real-time PCR. The results showed that 2850 bp of the duck IL-2 gene promoter region were obtained. There was one CpG island (-1231 bp/-902 bp) in which 11 CpG sites were distributed. The CpG1 and CpG2 sites are located between the binding sites of NFAT and AP-1, and they had higher homology methylation patterns in different individuals and tissues. The methylation frequencies of $28.5 \% \mathrm{CpG}$ sites showed negative correlations with the expression levels of the IL-2 mRNA, whereas $71.5 \%$ showed positive correlations. These results indicate that the transcription of duck $I L-2$ may be distinct from that of mammals. $C p G 1$ (-1284 bp) and CpG2 (-1264 bp) in the duck IL-2 promoter showed a higher homology of methylation patterns, indicating a similar regulatory effect on their gene expression, and these CpG sites may be essential for the regulation of transcription of duck $I L-2$. The methylation pattern of the IL-2 gene promoter in duck was tissue specific.
\end{abstract}

Key words: $C p G$, duck, DNA methylation, $I L-2$, mRNA expression, promoter.

(Centr Eur J Immunol 2018; 43 (4): 389-398)

\section{Introduction}

Interleukin 2 (IL-2), a cytokine, plays an important role in immune systems, and it can determine the differentiation and growth status of T cells, as well as enhancing their killing activities. Additionally, IL-2 induces the proliferation and antibody secretion of B cells and strengthens the killing activities of other types of immune cells, such as natural killer (NK) cells and monocytes [1-3].

The functions of IL-2 in immune processes have been clarified. However, little is known about the mechanism by which the expression of $I L-2$ is regulated in immune processes. As an important regulatory element in gene expression, the promoter can affect the transcription levels of genes and, accordingly, influence their functions. In the mouse and human $I L-2$ genes, the binding sites of many transcription factors have been identified, such as the nuclear factor of activated T cells (NFAT), nuclear factor $\mathrm{\kappa B}$ $(\mathrm{NF}-\kappa \mathrm{B})$, activating protein 1 (AP-1) families and the constitutive factors of the octamer-binding protein 1 (Oct-1).
These sites exist in the region of 300 bp upstream of the $I L-2$ gene transcription start site (TSS), and they may play essential roles in inducing $I L-2$ gene expression [4-6]. Rooney et al. (1995) showed that nucleotide mutations in the NFAT binding sites of the $I L-2$ promoter region completely block the anti-CD3-inducible promoter activity in transfected AE7 cells, indicating that the NFAT site is critical for regulating $I L-2$ transcription processes [7]. The increase of AP-1 activities in T cells stimulated with immobilized anti-CD3 antibodies enhances the expression and secretion of IL-2, suggesting that AP-1 is a major regulatory factor of $I L-2$ gene expression in splenic T cells activated through the TCR/CD3 complex [8]. Mutations in the AP-1 site significantly decrease or abrogate the induction of the $I L-2$ promoter [9]. Furthermore, NF- $\kappa$ B as an intracellular second messenger may play an important role in the transduction of signals from the outer cellular membranes, leading to IL-2-induced activation and growth via $\mathrm{NF}-\kappa \mathrm{B}[10]$.

Correspondence: Prof. Hehe Liu, Farm Animal Genetic Resources Exploration and Innovation Key Laboratory of Sichuan Province, Sichuan Agricultural University, Chengdu, Sichuan 611130, P.R. China, tel./fax: +86-028-86290985/+86-028-86290987, e-mail: Liuee1985@sicau.edu.cn

Submitted: 21.06.2016; Accepted: 10.01.2017 
As one of the most important epigenetic modification pathways, DNA methylation can affect gene transcription via the inactivation of positive or negative regulatory elements [11]. Studies showed that the methylation levels of the region consist of a $1.3 \mathrm{~kb}$ segment upstream of the TSS in the human $I L-2$ gene and contain $6 \mathrm{CpG}$ sites, which play an important role in inducing $I L-2$ gene expression [12]. Oct-1-binding followed by $\mathrm{CpG}$ demethylation is a key event in the epigenetic regulation of human $I L-2$ expression and significantly decreases expression of the $I L-2$ gene [13]. Similarly, methylation of the $I L-2$ promoter inhibited $I L-2$ transcription in T lymphocytes [14]. Effector CD4+ T cells stimulated with anti-CD3 and anti-CD28 resulted in a significant decrease of the average degree of methylation at each $\mathrm{CpG}$ site of the $I L-2$ promoter and enhancer, which leads to $I L-2$ transcription [15]. Therefore, the methylation level of the $I L-2$ promoter is important for $I L-2$ expression in both humans and mice.

The duck is an important agricultural economic animal with a greater ability to tolerate adversity than other poultry, indicating that the genetic background of the duck immune genes may differ from other species [16]. IL-2 plays an important role in immune systems and it is produced and secreted from the thymus, spleen and bursa of Fabricius [17-21]. Therefore, study of the transcriptional regulation of the $I L-2$ gene in the thymus, spleen and bursa of Fabricius is important for understanding the molecular basis of the regulation of the immune response in the duck. The objective of the present study was to ex- plore the characteristics of the duck $I L-2$ promoter, then through comparing and analyzing the relationship between IL-2 promoter methylation level and the expression level of mRNA in the spleen, thymus and bursa of Fabricius to research the possible effects of the methylation status on $I L-2$ gene expression in duck immune tissues. These studies may help us obtain insights into the major functions of IL-2 in immune processes and lay the foundation for future research on the transcriptional regulation of avian $I L-2$ genes in immune functions.

\section{Material and methods}

\section{Birds and sampling}

A total of 30 one-day-old ducklings of the NongHua-P strain were randomly selected from the Waterfowl Breeding Experimental Farm of Sichuan Agricultural University. All ducks were numbered, and blood was collected from the veins. After exsanguination, the tissues of the spleen, thymus and bursa of Fabricius were isolated. All samples were ground with liquid nitrogen and stored at $-80^{\circ} \mathrm{C}$ until needed. All procedures in the current study were approved by the Animal Welfare Committee of Sichuan province.

\section{Amplification and sequence analysis}

Total genomic DNA was extracted from the spleen, thymus and bursa of Fabricius with the DNA Extraction Kit (TianGen Co., Beijing, China) according to the man-

Table 1. Primers for amplification of duck $I L-2$ nucleotide sequence

\begin{tabular}{|c|c|c|c|}
\hline Primer name & $\begin{array}{l}\text { Primer sequence } \\
\left(5^{\prime}-3 \text { ') }\right.\end{array}$ & $\begin{array}{c}\text { Product } \\
\text { length (bp) }\end{array}$ & $\begin{array}{l}\mathrm{Tm} \\
\left({ }^{\circ} \mathrm{C}\right)\end{array}$ \\
\hline A1-F & CCCATAGAATAAGCCATAGAG & 745 & 56.5 \\
\hline A1-R & TATGCGACAGACGATGAA & & \\
\hline A2-F & CCCATAGAATAAGCCATAGAG & 829 & 53.9 \\
\hline A2-R & TATGCGACAGACGATGAA & & \\
\hline A3-F & CATTGCGTTTGATGGTGC & 1192 & 62.3 \\
\hline A3-R & GCTGGTTGGGCTGTGAT & & \\
\hline A4-F & GTTTTCAATGCTATTCGGTC & 1153 & 58.6 \\
\hline A4-R & ACTCTTGTCAGGTGGCGTA & & \\
\hline$\beta$-actin-F & GCTATGTCGCCCTGGATTTC & 168 & 60 \\
\hline$\beta$-actin-R & CACAGGACTCCATACCCAAGAA & & \\
\hline GAPDH-F & AAGGCTGAGAATGGGAAAC & 254 & 53 \\
\hline GAPDH-R & TTCAGGGACTTGTCATACTTC & & \\
\hline IL-2-F & GCATGAATGGGATTGATCTTGAG & 111 & 60 \\
\hline IL-2-R & CAATTTCTTCCTCCAAGGTGACTAT & & \\
\hline Mass ARRAY-F & AGGAAGAGAGAGATTGAGTTTTTTAGAAGTTGGGA & 414 & 62 \\
\hline Mass ARRAY-R & CAGTAATACGACTCACTATAGGGAGAAGGCTTAAACCAAATACTCCCCCTACTACC & & \\
\hline
\end{tabular}


Table 2. Software used for sequence analysis

\begin{tabular}{|c|c|c|}
\hline Name & Website & Application \\
\hline FPROM & $\begin{array}{l}\text { http://www.softberry.com/berry. } \\
\text { phtml?topic=fprom\&group=programs\&subgroup=promoter }\end{array}$ & Promoter prediction \\
\hline CpG Island Searcher & http://www.uscnorris.com/cpgislands2/cpg.aspx & CpG island analysis \\
\hline $\mathrm{PATCH}^{\mathrm{TM}}$ public 1.0 & $\begin{array}{l}\text { http://www.gene-regulation.com/cgi-bin/pub/programs/patch/ } \\
\text { bin/patch.cgi }\end{array}$ & Analysis of core transcription factor binding sites \\
\hline Plant Care & http://bioinformatics.psb.ugent.be/webtools/plantcare/html/ & Typical structural domain of promoter analysis \\
\hline
\end{tabular}

ufacturer's instructions. Four pairs of gene-specific primers (A1-A4, Table 1) were designed using the Primer 5.0 software (Premier Biosoft International, CA, USA) according to the predicted sequence of the $I L-2$ gene in NCBI (NW_004676716.1). PCR amplification was performed using duck gDNA, and the PCR products were cloned into the pMD19-T vector (TaKaRa Co., Dalian, China) after purification with the Gel Extraction Kit (OMEGA Co., Guangzhou, China). Finally, the products were transformed into competent DH5 alpha cells, screened for positive clones and propagated. The positive clones were then sent to a biological company (Invitrogen Co., Shanghai, China) for sequencing. The results were spliced using DNASTAR software (Madison, Wisconsin, USA). The characteristics of the $I L-2$ promoter and transcription factor binding sites were predicted and analyzed using the software provided. The software names, websites and applications are shown in Table 2.

\section{Mass ARRAY}

Quantitative DNA methylation analysis of IL-2 was performed using the Mass ARRAY Compact System. This system utilized mass spectrometry (MS) for the detection and quantitative analysis of DNA methylation using homogeneous mass CLEAVE (HMC) base-specific cleavage and matrix-assisted laser desorption/ionization time-of-flight MS (MALDI-TOF) [22]. Briefly, 3-5 $\mu$ g of genomic DNA was treated with sodium bisulfite and PCR amplified (Table 1). The bisulfite reactions were designed, which covered the region of interest, extending from $-1337 \mathrm{bp}$ to $-924 \mathrm{bp}$ relative to the translation initiation site. The reactions were in vitro transcribed and then cleaved by RNase A. The samples were quantitatively tested for DNA methylation status using matrix-assisted laser desorption ionization-time of flight mass spectrometry. Methylation data for the individual CpG sites or an aggregate of multiple $\mathrm{CpG}$ sites were generated with the EpiTyper v4.0 software (SEQUENOM, CA, USA). The nonapplicable reading frames and their corresponding sites were eliminated in the calculation.

\section{Real-time PCR}

Total RNA was extracted from the spleen, thymus and bursa of Fabricius of 15 ducklings with the RNA
Extraction Kit (TianGen Co, Beijing, China) according to the manufacturer's instructions, followed by synthesis of cDNA from $2 \mu \mathrm{g}$ of RNA using the PrimeScript RT Reagent Kit (TaKaRa Co., Dalian, China). The real-time PCR primers ( $I L-2$, Table 1$)$ were designed using the Primer 5.0 software (Premier Biosoft International, CA, USA) according to the mRNA sequence of the target gene IL-2 (JX239765.1), the endogenous reference gene $\beta$-actin (EF667345.1) and GAPDH (AY436595.1) of the duck in NCBI. The reaction was performed with a CFX96 RealTime PCR Detection System (Bio-Rad) using the reaction system SYBR Premix Reaction System Ex Taq II Kit (Takara, Dalian, China). The reaction program was $95^{\circ} \mathrm{C}$ for $30 \mathrm{~s}, 95^{\circ} \mathrm{C}$ for $5 \mathrm{~s}$, and $60^{\circ} \mathrm{C}$ for $30 \mathrm{~s}$, for 40 cycles. The dissolution curves were run from $65^{\circ} \mathrm{C}$ to $95^{\circ} \mathrm{C}$, reading for $10 \mathrm{~s}$ per $0.5^{\circ} \mathrm{C}$. Each sample was run in triplicate.

\section{Data analysis}

The $2^{-\Delta \Delta \mathrm{CT}}$ method [23] was employed to calculate the relative mRNA expression of the $I L-2$ gene according to the $\mathrm{Ct}$ value of PCR reaction, and the results were corrected using the internal control genes $\beta$-actin and GAPDH. All analyses were performed with the SAS software package, version 8.2 (SAS Institute, 1999). Results are formatted as means \pm standard error (SD). Matrix Hierarchical Cluster Analysis (normalized data, Euclidean distance, average linkage clustering) was obtained with the MultiExperiment Viewer (MeV) (http:// www.tm4.org/mev.html) (Dana-Farber Cancer Institute, 44 Binney St, Boston, MA, USA).

\section{Results \\ Cloning and analysis of duck $I L-2$ promoter sequence}

The duck $I L$-2 gene 5' flanking promoter region of $2850 \mathrm{bp}$ was obtained in this study. The potential promoter sequence and the TATA-box sites were predicted to exist in the 5' flanking sequences of the $I L-2$ gene in duck, chicken, human and mouse (Table 3). However, the position and number of the promoter elements in the duck $I L-2$ were different from those in chicken, human and mouse. The duck $I L-2$ gene promoter possessed two 
Table 3. Prediction of $I L-2$ gene promoter by FPROM software

\begin{tabular}{lcc}
\hline $\begin{array}{l}\text { Species } \\
\text { (Latin name) }\end{array}$ & $\begin{array}{c}\text { Promoter position } \\
\text { (bp) }\end{array}$ & $\begin{array}{c}\text { TATA box position } \\
\text { (bp) }\end{array}$ \\
\hline $\begin{array}{l}\text { Duck } \\
\text { (Anas platyrhynchos) }\end{array}$ & -2152 & -2180 \\
\hline & -996 & -1027 \\
\hline $\begin{array}{l}\text { Human } \\
\text { (Homo sapiens) }\end{array}$ & -2026 & -2056 \\
\hline $\begin{array}{l}\text { House mouse } \\
\text { (Mus musculus) }\end{array}$ & -1391 & -1421 \\
\hline $\begin{array}{l}\text { Chicken } \\
\text { (Gallus gallus) }\end{array}$ & -1875 & -1905 \\
\hline & -526 & -555
\end{tabular}

The human IL-2 promoter sequence, house mouse IL-2 and chicken IL-2 promoter sequence were from Homo sapiens genome (NC_000004.12), Mus musculus genome (NC_000069.6) and Gallus gallus genome (NC_006091.3), respectively.

TATA-box elements, located at $-2180 \mathrm{bp}$ and $-1027 \mathrm{bp}$. By contrast, there was only one TATA-box in the $I L-2$ promoter of human and mouse, located at -2056 bp and $-1421 \mathrm{bp}$, respectively. In addition, although the chicken IL-2 promoter also had two TATA-box elements, their positions were different from duck, located at -1905 bp and $-555 \mathrm{bp}$ in the promoter.

The bioinformatics results revealed that the duck IL-2 promoter region contains numerous transcription factor binding sites, a core-promoter structure TATA-box and two typical CpG islands (Fig. 1A). The region from $-900 \mathrm{bp}$ to -1500 bp upstream of the duck IL-2 transcription start site was possibly the core transcription regulation region. In this region, there was NF- $\kappa \mathrm{B}, \mathrm{AP}-1$, NFAT, Sp-1 and two TATA-box elements. Moreover, the predicted $\mathrm{CpG}$ islands in the IL-2 promoter region showed that the transcription of IL-2 may be influenced by epigenetic modifications (Fig. 1B). There were $11 \mathrm{CpG}$ sites in the $\mathrm{CpG}$ island (-1231 bp/-902 bp); However, only 7 had methylation information. $\mathrm{CpG} 1$ and $\mathrm{CpG} 2$ are located between NFAT and AP-1, and only CpG4 was located in the binding site of the transcription factor GATA-1. Moreover, there are no additional $\mathrm{CpG}$ sites located in the transcription factor binding sites (Fig. 1C).

\section{Cluster analysis for methylation levels of CpG sites}

The two-way cluster results of the methylation states in the $\mathrm{CpG}$ islands of the duck $I L-2$ promoter are listed in Fig. 2. The data showed that most individuals have the highest homology distributed in the $\mathrm{CpG}$ island site methylation states in spleen tissues and have relatively higher homology distributed in the thymus and bursa. The data indicate that the methylation patterns of the duck $I L-2$ promoter $\mathrm{CpG}$ locus were tissue specific, and the spleen had a distinct methylation pattern of the $I L-2$ promoter island compared to the thymus and bursa. Additionally, the sites of CpG1 and CpG2 in different individuals and tissues had higher homology methylation patterns. There was likely a similar regulatory effect among these cluster sites for the methylation patterns on $I L-2$ gene transcription.

\section{Comparison of CpG site methylation levels in immune tissues}

Figure 3 is a comparison of methylation frequency of different $\mathrm{CpG}$ sites in the same tissue. The results show that the $\mathrm{CpG} 2$ site methylation frequency was significantly higher than that of other sites in the thymus, spleen and bursa of Fabricius $(p<0.05)$. The methylation frequency of CpG1, CpG4, CpG7 and CpG9 was significantly higher than that of $\mathrm{CpG6}$ in the thymus and bursa of Fabricius $(p<0.05)$. However, the CpG6 site methylation frequency was significantly higher than that of other sites except the $\mathrm{CpG} 2$ site in the spleen $(p<0.05)$. These data indicate that $\mathrm{CpG} 2$ methylation frequency was significantly higher than that of other sites in the thymus, spleen and bursa of Fabricius $(p<0.05)$, and the methylation pattern of the CpG6 site in the spleen was different from the thymus and bursa of Fabricius.

\section{The impact of promoter methylation states on IL-2 transcription}

Figure $4 \mathrm{~A}$ is a schematic diagram of the regulatory elements within the duck $I L-2$ promoter. It lists a $1.5 \mathrm{~kb}$ nucleotide sequence upstream from the TSS, which contains $7 \mathrm{CpG}$ sites. The results showed that the methylation levels at the same $\mathrm{CpG}$ site among tissues were clearly different (Fig. 4B). Specifically, the methylation frequency of CpG1, CpG9 and CpG10 in the thymus and bursa of Fabricius was significantly higher than in the spleen $(p<0.05)$. Additionally, the methylation frequency of the $\mathrm{CpG} 2$ site in the thymus was significantly higher than in the spleen and bursa of Fabricius $(p<0.05)$, and the methylation frequency of CpG6 in the spleen was significantly higher than in the thymus and bursa of Fabricius $(p<0.05)$. The overall methylation states and mRNA expression of $I L-2$ among tissues are compared in Fig. 4C, D and show that the overall methylation states in the thymus were significantly higher than in the spleen $(p<$ 0.05) (Fig. 4C). The expression of $I L-2$ mRNA in the bursa of Fabricius was higher than in the spleen, and that in the spleen was higher than in the thymus. However, there were no significant differences in $I L-2$ mRNA expression among the three immune tissues (Fig. 4D).

\section{Correlation analysis between promoter methylation states and expression levels of IL-2}

The correlation analysis showed that there were no significant correlations between the methylation states of each 
A

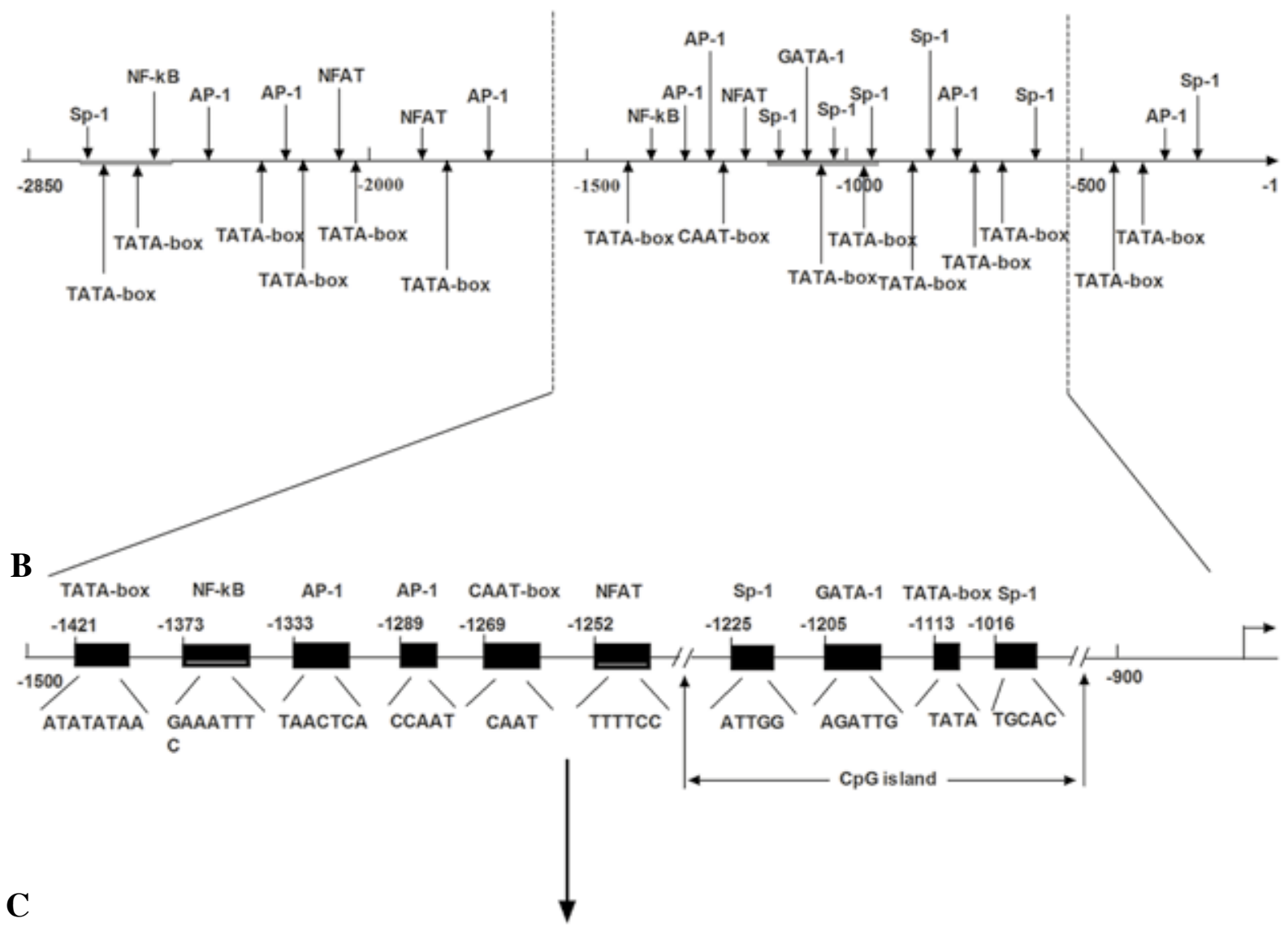

-1499 GTATTGGGCAAACTTTAGTCAACGATAGTTCTCTGTTTTCTATTTCACGGTCCTTTCCAA

-1439 CGTTTGACGTACAGAT GATATATAACGTTCGAT GTT GTCGATGATTCTGAAGGCACACTG

-1379 ACATAAGAAATTTACGAAT AT GAAT AACGT TTT GGTCCTTT AÇT TCTAACTCAAGAGGTC

-1319 TTCGACCCT AGT ATGACGATT AATT GCGGTCCAATT ACCAGACGCCT GTCAATITTGTCTG

NFAT

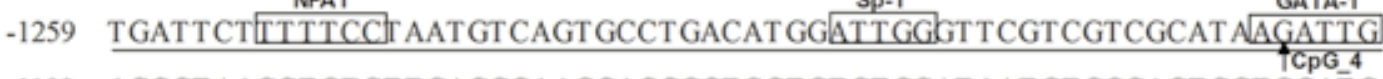

-1199 ACCGTAACCTGTCTTGACGGAAGGAGGGGTCGTGT GT GCATAAT GT GCCAGTCGT GGAT G

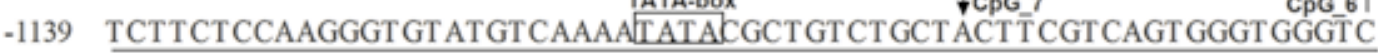

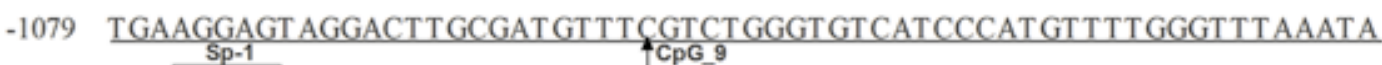

-1019 GGGTGCACCGAAGTCGTCGTTTTACGTACTTCGTCTTCGTTACACACAAGT GTATTACAA

-959 TGCCTGAAGACCCGTC GTCCCCCTCGTAAACCGAGT CTT GACGT GAGTCACTTCTTTTGC

-899 TAGATAAAATTT GTAACGAGT GT CGTAAGGGT TCT AATAGCCCGTCGTCACGAAACT TAG

-839 TCCTTTCTCTGTGTCAGGTCATCATGACACGCTCTCAGGTTACAGTTTACTGACGAATGA

Fig. 1. Schematic representation of $I L-2$ promoter showing the regulatory elements governing gene transcription in duck. A) Regulatory elements predicted in the duck $I L-2$ promoter. The arrow indicates the position of the core transcription factor binding sites and TATA-boxes, and the thick gray line shows the $\mathrm{CpG}$ islands. B) Transcriptional regulatory region. C) $\mathrm{CpG}$ sites in the transcriptional regulatory region. There are $11 \mathrm{CpG}$ sites in this region, of which CpG3, CpG5, CpG8 and CpG11 site had no methylation data. The bases of the arrow points are CpG sites. The transcription factor binding site and the promoter structure are represented by the base sequence in the box, and the underlined sequence is the methylation detection region 


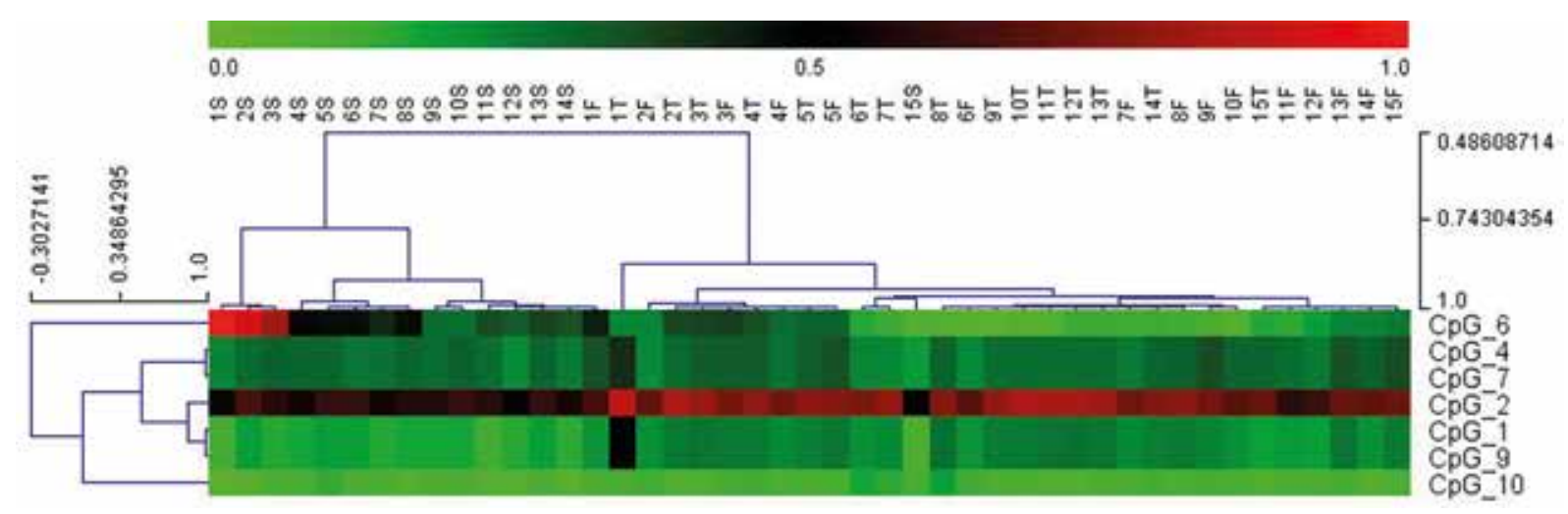

Fig. 2. Cluster analysis of tissues and CpG sites by $I L-2$ methylation in immune tissues. Light green to deep red represents the frequency of methylation from 0 to $100 \%$. Each row represents 1 sample, and each column represents 1 methylation site. Seven CpG sites of the $I L-2$ promoter were analyzed by cluster analysis in the thymus $(n=15), \operatorname{spleen}(n=15)$ and bursa of Fabricius $(n=15)$. The molecular weights of CpG1 and 9, 4 and 7 sites are the same, and the methylation of each site is the mean value. T represents the thymus, $\mathrm{S}$ represents the spleen, and $\mathrm{F}$ represents the bursa of Fabricius

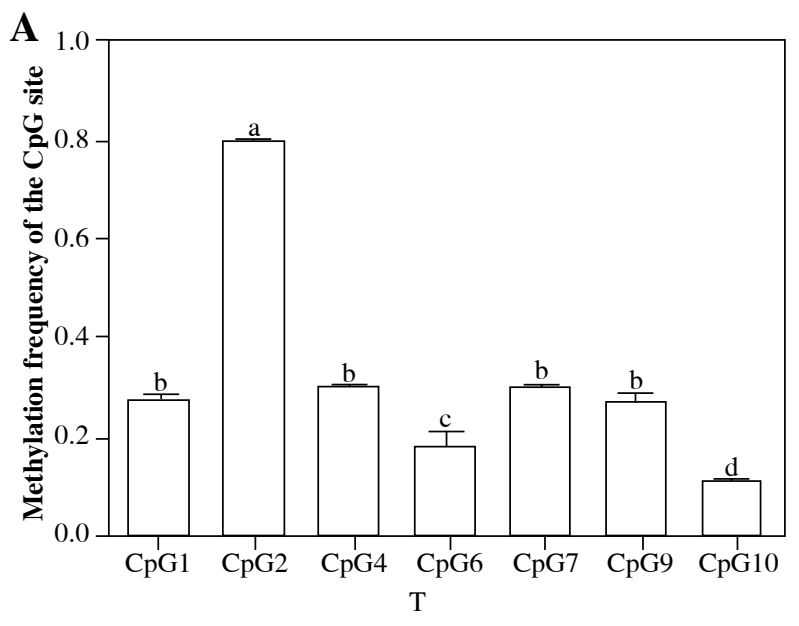

C

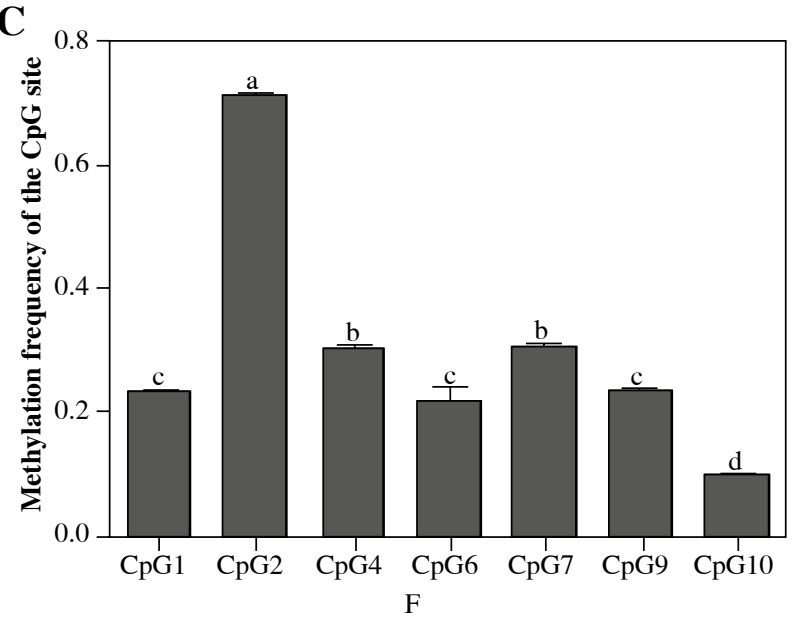

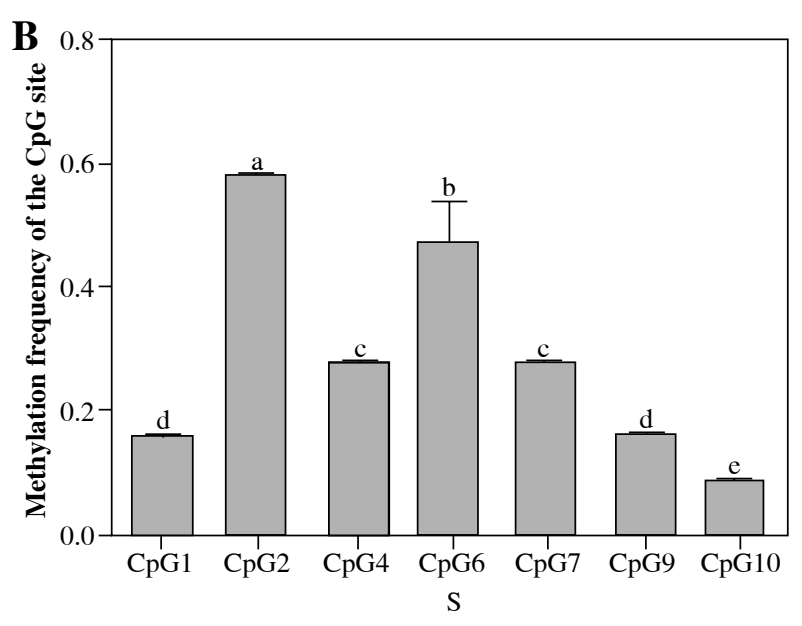

Fig. 3. Comparison of $\mathrm{CpG}$ site methylation levels of IL-2 in the thymus, spleen and bursa of Fabricius. A) Each CpG site's methylation level in the thymus $(n=15)$. B) Each CpG site's methylation level in the spleen $(n=15)$. C) Each CpG site's methylation level in the bursa of Fabricius $(n=15)$. T represents the thymus, $\mathrm{S}$ represents the spleen, and $\mathrm{F}$ represents the bursa of Fabricius. The same letter indicates no significant differences $(p>0.05)$, and different letters indicate significant differences $(p<0.05)$. $\mathrm{T}$ represents the thymus, $\mathrm{S}$ represents the spleen, and $F$ represents the bursa of Fabricius. The same letter indicates no significant differences $(p>0.05)$, and different letters indicate significant differences $(p<0.05)$ 


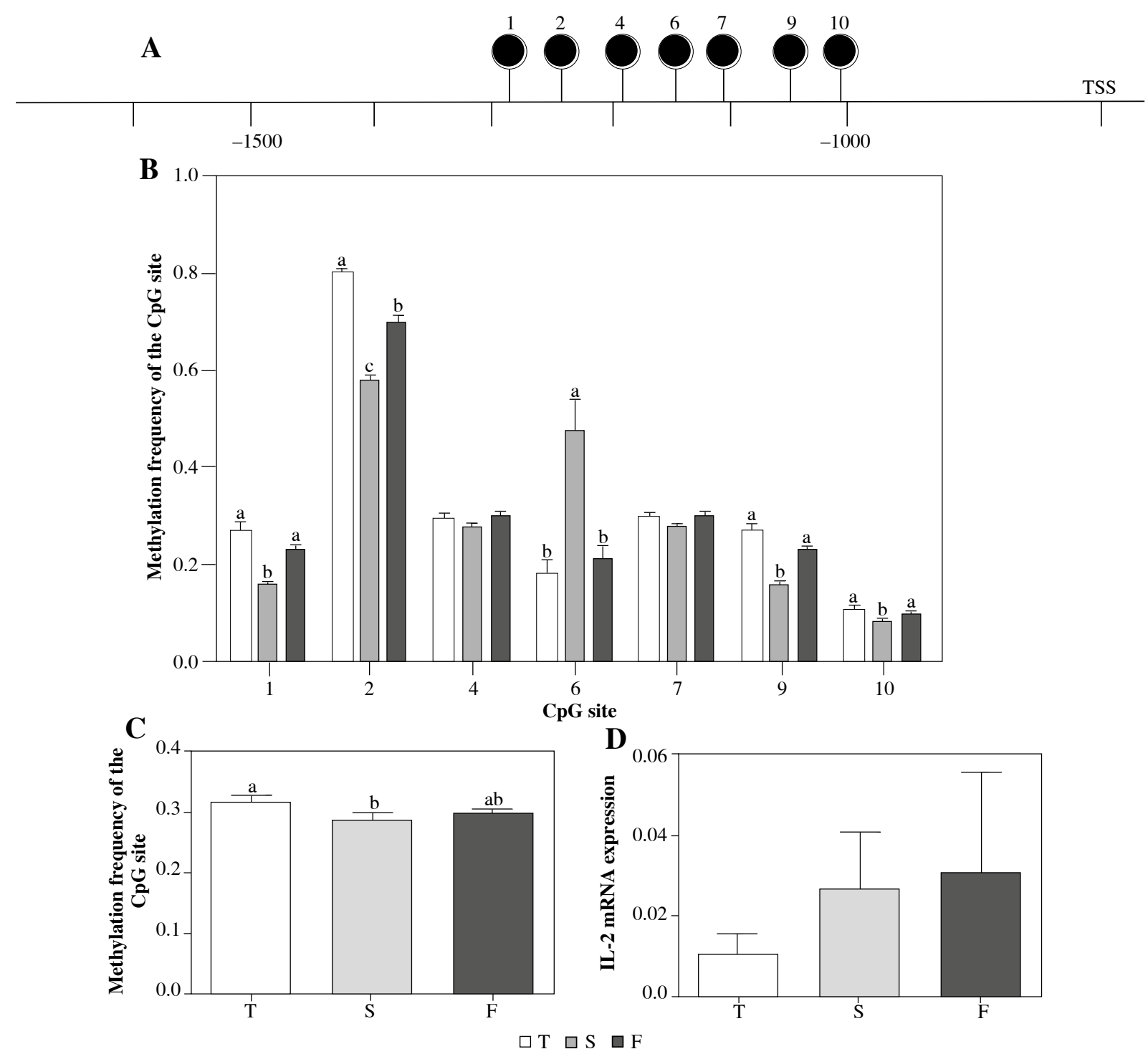

Fig. 4. Comparison between promoter methylation states and mRNA expression of $I L-2$ in immune tissues. A) Schematic diagram of the regulatory elements within the duck $I L-2$ promoter. The region shown includes $1.5 \mathrm{~kb}$ upstream from the TSS, which contains $7 \mathrm{CpG}$ sites $(\bullet$, methylated CpG). B) Methylation frequency of CpG sites of the duck $I L-2$ gene promoter in the spleen, thymus and bursa of Fabricius $(n=15)$. C) Overall methylation frequency of $I L-2$ gene promoter in the spleen, thymus and bursa of Fabricius $(n=15)$. D) mRNA expression of $I L-2$ gene in the spleen, thymus and bursa of Fabricius. T represents the thymus, S represents the spleen, and F represents the bursa of Fabricius. The same letter indicates no significant differences $(p>0.05)$, and different letters indicate significant differences $(p<0.05)$

Table 4. Correlation analysis between methylation status of $\mathrm{CpG}$ sites and mRNA expression

\begin{tabular}{cccccccccc}
\hline & CpG1 & CpG2 & CpG4 & CpG6 & CpG7 & CpG9 & CpG10 & Overall \\
\hline $\mathrm{T}$ & -0.025 & 0.000 & 0.028 & -0.007 & 0.028 & -0.025 & -0.151 & -0.068 \\
& $(0.931)$ & $(0.999)$ & $(0.925)$ & $(0.981)$ & $(0.925)$ & $(0.931)$ & $(0.607)$ & $(0.809)$ \\
\hline $\mathrm{S}$ & 0.171 & 0.29 & 0.352 & -0.062 & 0.352 & 0.171 & -0.129 & 0.106 \\
& $(0.543)$ & $(0.294)$ & $(0.198)$ & $(0.826)$ & $(0.198)$ & $(0.543)$ & $(0.646)$ & $(0.707)$ \\
\hline $\mathrm{F}$ & 0.443 & 0.380 & 0.295 & 0.210 & 0.295 & 0.443 & 0.023 & 0.406 \\
& $(0.098)$ & $(0.163)$ & $(0.286)$ & $(0.453)$ & $(0.286)$ & $(0.098)$ & $(0.935)$ & $(0.133)$ \\
\hline
\end{tabular}

Trepresents the thymus, $S$ represents the spleen, and $F$ represents the bursa of Fabricius. The molecular weights of CpG1 and 9,4 and 7 sites were similar; therefore the methylation of each site was given as their mean value. $P$ values are in parentheses. $N=15$ 
CpG site and IL-2 mRNA expression in the thymus, spleen and bursa of Fabricius (Table 4). We found that $28.5 \%$ of the $\mathrm{CpG}$ sites showed negative correlations between the methylation frequencies and the mRNA expression of IL-2, and $71.5 \%$ showed positive correlations. The methylation levels of CpG1, CpG6 and CpG10 in the thymus and CpG6 and CpG10 in the spleen were negatively correlated with the expression of IL-2 $(p>0.05)$. The other CpG sites were positively correlated with the expression of IL-2 mRNA in these tissues $(p>0.05)$.

\section{Discussion}

Interleukin 2, which is mainly produced in the thymus and spleen, plays an important role in immune systems. The functions of IL-2 in immune processes have been clarified. However, the mechanism by which the expression of $I L-2$ is regulated in immune processes has rarely been studied in birds. In addition, it had been reported that the passively acquired antibodies are released into the circulation when the yolk is digested by the embryo and can be detected as early as the $11^{\text {th }}$ day of incubation, and IL2-producing cells such as those of the thymus and spleen can be detected in the late embryo [24-26], suggesting that $I L-2$ expression may be triggered by stimulation of the embryonic period in immune tissues of nadve newborn (one-day-old) ducklings. On the other hand, stimulation of human CD4+ T cells induced $I L-2$ expression following epigenetic changes, including active demethylation of specific $\mathrm{CpG}$ sites, as shown in previous documents. Therefore, to minimize the influence of external factors on the methylation status, the immune tissues of nadve newborn (one-day-old) ducklings were selected to study. In this study, the duck $I L-2$ gene 5' flanking promoter region of $2850 \mathrm{bp}$ was obtained. It was predicted that there was one NF- $\kappa \mathrm{B}$, two AP-1, one NFAT, and other core transcription factor binding sites distributed from $-900 \mathrm{bp}$ to $-1500 \mathrm{bp}$, upstream of the duck $I L-2$ transcription start site (Fig. 1B). In addition, there were two TATA-boxes and one CAAT-box typical core promoter structure. The NFAT family, AP-1 and NF- $\kappa$ B are important transcription factors inducing $I L-2$ transcription in humans and mice, and all regulate the transcription of $I L-2$ [27-31]. The data indicate that this region may play a key role in regulating $I L-2$ transcription. Studies in mice and humans revealed that $300 \mathrm{bp}$ upstream of the transcription start site of the $I L-2$ gene was the core regulatory region [5], revealing that the transcriptional regulation of the $I L-2$ promoter in ducks may be different from that in mammals. Furthermore, the duck $I L-2$ promoter had two CpG islands, of which one $(-1231 \mathrm{bp} /$ $-902 \mathrm{bp}$ ) is located in the core of the transcribed region (Fig. 1B). This indicates that duck $I L-2$ gene transcription is possibly regulated by methylation modification and distinct from that of mammals.

The epigenetic modification of a gene promoter is an important method of regulating gene transcription. As a main type of epigenetic modification, DNA methylation plays an important role in the transcription of genes. Several studies have indicated that methylation of promoters inhibits the transcription of genes by blocking transcription factor binding to its promoter regions [32-34]. In the present study, the CpG island (-1231 bp/-902 bp) was important for the gene expression of duck $I L-2$ because there were more transcription factors predicted to exist in this area. Therefore, the $\mathrm{CpG}$ sites were detected in this region, and the relative position of the $\mathrm{CpG}$ sites and the core transcription factor binding sites were analyzed. The results showed that there were $11 \mathrm{CpG}$ sites distributed in the CpG island (-1231 bp/-902 bp). Furthermore, CpG1 and CpG2 were located between NFAT and AP-1 (Fig. 1C), and $\mathrm{CpG} 2$ methylation frequency was significantly higher than that of other sites in the thymus, spleen and bursa of Fabricius $(p<0.05)$ (Fig. 3). In addition, we found that $\mathrm{CpG} 1$ and $\mathrm{CpG} 2$ in different individuals and tissues had higher homology methylation patterns (Fig. 2), suggesting that there was a similar regulation effect among these cluster sites for the effects of methylation patterns on $I L$-2 gene transcription. Some studies have confirmed that cooperative interactions between NFAT and AP-1 were essential for $I L-2$ gene expression induction $[35,36]$. Therefore, our data suggest that these $\mathrm{CpG}$ sites may be essential for the regulation of transcription of duck $I L-2$.

Further studies have been performed to assess the effect of DNA methylation on the expression of $I L-2$ in duck immune tissues. First, a two-way cluster analysis was used to determine the methylation frequency of the $7 \mathrm{CpG}$ sites of the $I L-2$ promoter in the thymus, spleen and bursa of $\mathrm{Fa}-$ bricius. The results revealed that the methylation level of the $I L-2$ gene in the spleen tissue was different from the thymus and bursa of Fabricius (Fig. 2), suggesting that the methylation pattern of the $I L-2$ gene promoter in the duck was tissue specific. Similar results were reported by Ma et al., who found that the degree of DNA methylation differed between porcine fat and muscle tissue in pigs [37]. Fan et al. found that the frequency of methylation is different in human tissues [38]. This may be because the methylation pattern of the CpG6 site was different from the thymus and the bursa of Fabricius, leading to the methylation pattern of the $I L-2$ gene in the spleen tissue being different from the thymus and bursa of Fabricius. Second, the correlation of $I L-2$ promoter methylation states and the levels of $I L-2$ gene expression were analyzed. The results showed that the expression of $I L-2$ mRNA was different in the thymus, spleen and bursa of Fabricius in Fig. 4D $(p>0.05)$. A study indicated that the strength of the immune response is regulated largely by the amount of IL-2 available for T-cell growth [39]. Therefore, these results suggested that the strength of the immune response may be different in young duck immune tissue. This conclusion was in agreement with a previous study by Longenecker et al. (1966), who reported that the immune function of the thymus was weak. In chicken, 
the spleen is of primary importance in the development of immunity to this strain of Plasmodium lophurae, the bursa is of secondary importance and contributes something to the development of the immune mechanism before 18 days of age [40], and there were differences in the immune function of the thymus and bursa of Fabricius [41]. In addition, the results showed that $28.5 \%$ of the $\mathrm{CpG}$ sites showed negative correlations between the methylation frequencies and the mRNA expression of $I L-2$, and $71.5 \%$ showed positive correlations (Table 4). For the methylation level of all $\mathrm{CpG}$ sites, the methylation level in the thymus was significantly higher than that in the spleen $(p<0.05)$ (Fig. 4C). Moreover, the expression of $I L-2$ mRNA in the spleen was higher than that of the thymus $(p>0.05)$. These data show that the methylation frequency may be negatively correlated with expression of the IL-2 gene in duck immune tissue. The conclusion is consistent with the study by Marie-Chloé et al., who found that arginine methylation of Tat negatively regulates its transactivation activity [42]. The DNA methylation level of the Six 1 promoter core region was negatively correlated with Sixl gene expression in vivo [43]. However, whether DNA methylation has an effect on the expression of duck IL-2 has to be determined by some in vitro transcription experiments such as reporter plasmids carrying $I L-2$ promoter/enhancer region or mutants, or using the promoter-targeted shRNAi method under stimulation conditions.

\section{Conclusions}

The duck $I L$-2 gene 5' flanking promoter region of $2850 \mathrm{bp}$ was obtained in this study. A total of $11 \mathrm{CpG}$ sites were predicted in the $\mathrm{CpG}$ island (-1231 bp/-902 bp) of the duck $I L-2$ promoter. The transcription of duck $I L-2$ may be distinct from that of mammals. CpG1 (-1284 bp) and CpG2 (-1264 bp) in the duck $I L-2$ promoter showed a higher homology of methylation patterns, indicating a similar regulatory effect on their gene expression, and these CpG sites may be essential for the regulation of transcription of duck $I L-2$. The methylation pattern of the $I L-2$ gene promoter in the duck was tissue specific.

\section{Acknowledgments}

This work was supported by the application foundation project of Sichuan Science and Technology Bureau (2015JY0110), the National Natural Science Foundation of China (No. 31301964), Chinese Agriculture Research Service (No. CARS-43-6), the Major Project of Sichuan Education Department (13ZA0252), and the Breeding of Multiple Crossbreeding Systems in Waterfowl (2011NZ0099-8).

The authors declare no conflict of interest.

\section{References}

1. Hefeneider SH, Conlon PJ, Henney CS, et al. (1983): In vivo interleukin 2 administration augments the generation of alloreactive cytolytic $\mathrm{T}$ lymphocytes and resident natural killer cells. J Immunol 130: 222-227.

2. Merluzzi VJ, Welte K, Savage DM, et al. (1983): Expansion of cyclophosphamide-resistant cytotoxic precursors in vitro and in vivo by purified human interleukin 2 . J Immunol 131: 806-809.

3. Malek TR (2003): The main function of IL-2 is to promote the development of T regulatory cells. J Leukoc Biol 74: 961-965.

4. Crabtree GR, Clipstone NA (1994): Signal transmission between the plasma membrane and nucleus of T lymphocytes. Annu Rev Biochem 63: 1045-1083.

5. Garrity PA, Chen D, Rothenberg EV, et al. (1994): Interleukin-2 transcription is regulated in vivo at the level of coordinated binding of both constitutive and regulated factors. Mol Cell Biol 14: 2159-2169.

6. Jain J, Loh C, Rao A (1995): Transcriptional regulation of the IL-2 gene. Curr Opin Immunol 7: 333-342.

7. Rooney JW, Sun YL, Glimcher LH, et al. (1995): Novel NFAT sites that mediate activation of the interleukin-2 promoter in response to T-cell receptor stimulation. Mol Cell Biol 15: 6299-6310.

8. Ochi Y, Koizumi T, Kobayashi S, et al. (1994): Analysis of IL-2 gene regulation in c-fos transgenic mice. Evidence for an enhancement of IL-2 expression in splenic T cells stimulated via TCR/CD3 complex. J Immunol 153: 3485-3490.

9. Jain J, Valge-Archer VE, Rao A (1992): Analysis of the AP-1 sites in the IL-2 promoter. J Immunol 148: 1240-1250.

10. Arima N, Kuziel WA, Grdina TA, et al. (1992): IL-2-induced signal transduction involves the activation of nuclear NF-kappa B expression. J Immunol 149: 83-91.

11. Jones PA, Takai D (2001): The role of DNA methylation in mammalian epigenetics. Science 293: 1068-1070.

12. Crispin JC, Tsokos GC (2009): Transcriptional regulation of IL-2 in health and autoimmunity. Autoimmun Rev 8: 190-195.

13. Murayama A, Sakura K, Nakama M, et al. (2006): A specific $\mathrm{CpG}$ site demethylation in the human interleukin 2 gene promoter is an epigenetic memory. EMBO J 25: 1081-1092.

14. Bruniquel D, Schwartz RH (2003): Selective, stable demethylation of the interleukin-2 gene enhances transcription by an active process. Nat Immunol 4: 235-240.

15. Thomas RM, Gao L, Wells AD (2005): Signals from CD28 induce stable epigenetic modification of the IL-2 promoter. J Immunol 174: 4639-4646.

16. Huang Y, Li Y, Burt DW, et al. (2013): The duck genome and transcriptome provide insight into an avian influenza virus reservoir species. Nat Genet 45: 776-783.

17. Hoffmann-Fezer G, Rodt H, Gotze D, et al. (1977): Anatomical distribution of $\mathrm{T}$ and $\mathrm{B}$ lymphocytes identified by immunohistochemistry in the chicken spleen. Int Arch Allergy Appl Immunol 55: 86-95.

18. Seto F (1981): Early development of the avian immune system. Poult Sci 60: 1981-1995.

19. Emilie D, Crevon MC, Cohen-Kaminsky S, et al. (1991): In situ production of interleukins in hyperplastic thymus from myasthenia gravis patients. Hum Pathol 22: 461-468.

20. Smith KA (1988): Interleukin-2: inception, impact, and implications. Science 240: 1169-1176.

21. Liu X, Li Z, Han C, et al. (2012): Effects of dietary manganese on $\mathrm{Cu}, \mathrm{Fe}, \mathrm{Zn}, \mathrm{Ca}, \mathrm{Se}$, IL- $1 \beta$, and IL-2 changes of immune organs in cocks. Biol Trace Elem Res 148: 336-344. 
22. Ehrich M, Nelson MR, Stanssens P, et al. (2005): Quantitative high-throughput analysis of DNA methylation patterns by base-specific cleavage and mass spectrometry. Proc Natl Acad Sci U S A 102: 15785-15790.

23. Schmittgen TD, Livak KJ (2008): Analyzing real-time PCR data by the comparative $\mathrm{C}(\mathrm{T})$ method. Nat Protoc 3: 11011108.

24. Seto F (1981): Early development of the avian immune system. Poult Sci 60: 1981-1995.

25. Emilie D, Crevon MC, Cohenkaminsky S, et al. (1991): In situ production of interleukins in hyperplastic thymus from myasthenia gravis patients. Hum Pathol 22: 461-468.

26. Hoffmann-Fezer G, Rodt H, Götze D, et al. (1977): Anatomical distribution of $\mathrm{T}$ and $\mathrm{B}$ lymphocytes identified by immunohistochemistry in the chicken spleen. Int Arch Allergy Appl Immunol 55: 86-95.

27. Peng SL, Gerth AJ, Ranger AM, et al. (2001): NFATc1 and NFATc2 together control both $\mathrm{T}$ and $\mathrm{B}$ cell activation and differentiation. Immunity 14: 13-20.

28. Ranger AM, Oukka M, Rengarajan J, et al. (1998): Inhibitory function of two NFAT family members in lymphoid homeostasis and Th2 development. Immunity 9: 627-635.

29. Paliogianni F, Raptis A, Ahuja SS, et al. (1993): Negative transcriptional regulation of human interleukin 2 (IL-2) gene by glucocorticoids through interference with nuclear transcription factors AP-1 and NF-AT. J Clin Invest 91: 14811489.

30. Shapiro VS, Truitt KE, Imboden JB, et al. (1997): CD28 mediates transcriptional upregulation of the interleukin-2 (IL-2) promoter through a composite element containing the CD28RE and NF-IL-2B AP-1 sites. Mol Cell Biol 17: 40514058.

31. Ghosh S, May MJ, Kopp EB (1998): NF-кB and Rel proteins: evolutionarily conserved mediators of immune responses. Annu Rev Immunol 16: 225-260.

32. Crider KS, Yang TP, Berry RJ, et al. (2012): Folate and DNA methylation: a review of molecular mechanisms and the evidence for folate's role. Adv Nutr 3: 21-38.

33. Tate PH, Bird AP (1993): Effects of DNA methylation on DNA-binding proteins and gene expression. Curr Opin Genet Dev 3: 226-231.

34. Boyes J, Bird A (1991): DNA methylation inhibits transcription indirectly via a methyl-CpG binding protein. Cell 64 : 1123-1134.

35. Boise LH, Petryniak B, Mao X, et al. (1993): The NFAT-1 DNA binding complex in activated T cells contains Fra-1 and JunB. Mol Cell Biol 13: 1911-1919.

36. Northrop JP, Ho SN, Chen L, et al. (1994): NF-AT components define a family of transcription factors targeted in T-cell activation. Nature 369: 497-502.

37. Ma JD, Li MZ, Zhou SL, et al. (2012): Methylation-sensitive amplification polymorphism analysis of fat and muscle tissues in pigs. Genet Mol Res 11: 3505-3510.

38. Fan S, Zhang X (2009): CpG island methylation pattern in different human tissues and its correlation with gene expression. Biochem Biophys Res Commun 383: 421-425.

39. Efrat S, Kaempfer R (1984): Control of biologically active interleukin 2 messenger RNA formation in induced human lymphocytes. Proc Natl Acad Sci U S A 81: 2601-2605.

40. Longenecker BM, Breitenbach RP, Farmer JN (1966): The role of the bursa Fabricius, spleen and thymus in the control of a Plasmodium lophurae infection in the chicken. J Immunol 97: 594-599.
41. Warner N, Szenberg A (1964): The immunological function of the bursa of Fabricius in the chicken. Ann Rev Microbiol 18: 253-266.

42. Marie-Chloé B, Chen L, Russell RS, et al. (2005): Methylation of Tat by PRMT6 regulates human immunodeficiency virus type 1 gene expression. J Virol 79: 124-131.

43. Wu W, Ren Z, Liu H, et al. (2013): Core promoter analysis of porcine Six 1 gene and its regulation of the promoter activity by CpG methylation. Gene 529: 238-244. 